\title{
Teknokultura
}

ISSNe: 1549-2230

http://dx.doi.org/10.5209/TEKN.59854

\section{Fabricación digital y arquitectura como herramienta de concienciación social. REme: Refugio para situaciones de emergencia ${ }^{1}$}

\author{
Juan Carlos Castro Domínguez²; Yordhana Gómez ${ }^{3}$; Francisco de Juanes ${ }^{4}$; Leandro Moreno ${ }^{5}$
}

Recibido: 10 de abril de 2018 / Aceptado: 5 de septiembre de 2018 Open peer reviews

Resumen. Este artículo muestra cómo un espacio arquitectónico, como es un refugio temporal, se puede convertir en una herramienta de comunicación y concienciación muy potente. Así, los factores estrictamente arquitectónicos como son el sistema constructivo, su capacidad espacial, estética o su capacidad para albergar diferentes usos, son agentes de mediación de gran valor y potencia divulgativa. Es decir, la estructura arquitectónica amplía su misión y se convierte en un medio de comunicación de valores y puntos de vista, ampliando su capacidad comunicadora. De esta manera, a través de acciones concretas de comunicación BTL (Bellow The Line) la arquitectura se convierte en centro de la acción comunicativa.

Palabras clave: fab lab; empoderamiento social; refugio temporal; técnicas BTL.

\section{[en] Digital fabrication and architecture as a social awareness tool. REme: emergency shelter.}

\begin{abstract}
This paper focuses on demonstrating how an architectural space, such as a shelter, can be used as a communication and awareness powerful tool. Architectural factors as the construction system, its spatiality, its aesthetics or its different programs, are agents of mediation of great value and informative power. Then, we can say that architectonic structures can be a wide media that contribute with new values and points of view.
\end{abstract}

Keywords: BTL techniques; fab lab; shelter; social empowerment.

Sumario. 1. Introducción. 2. \#REme. 3. Mundo Fab Lab y fabricación digital. 4. Ensayo. 5. Arquitectura para la comunicación, semiótica y diseño. 6. Conclusiones. 7. Referencias.

Cómo citar: Castro Domínguez, J. C.; Gómez, Y.; De Juanes, F.; Moreno, L. (2018). Fabricación digital y arquitectura como herramienta de concienciación social. REme: Refugio para situaciones de emergencia, en Teknokultura 15(2), 387-398.

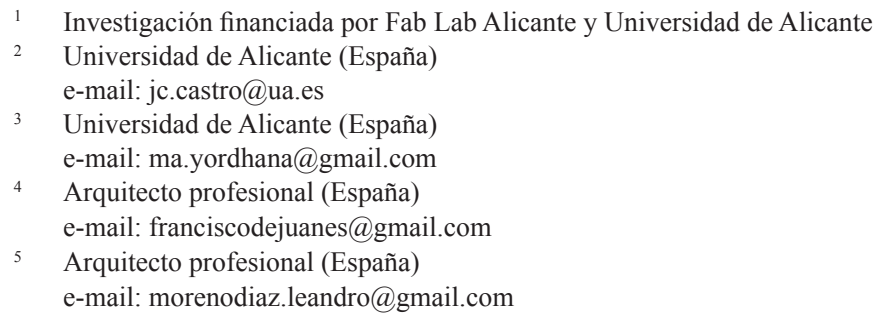




\section{Introducción}

\#REme es un prototipo de refugio temporal para situaciones de emergencia, ideado y desarrollado en Fab Lab Alicante. El objetivo principal del proyecto es desarrollar un espacio habitacional temporal que sea fácil de construir con técnicas y herramientas propias de la fabricación digital. Este objetivo responde a las características propias de este tipo de modelos, a la proliferación de laboratorios de fabricación digital y a la situación a la que se destina. En este proyecto se exploran las filosofías de conocimiento libre y DIY (Do It Yourself), incorporando el diseño paramétrico como herramienta que permite una mayor versatilidad y adaptabilidad a las diferentes situaciones en las que se pudiera utilizar. El diseño de \#REme se basa en la repetición de una unidad estructural, construida a partir de pequeñas piezas susceptibles de ser mecanizadas en cualquier taller de pequeña escala que disponga de máquinas de control numérico (CNC) o corte láser. Se trata de un prototipo susceptible de ser modificado, versátil tanto en materiales como en posibilidades de producción. Su diseño y fabricación en código abierto, basado en materiales comunes y diseñados a partir de una estructura geométrica clara y sencilla permite reprogramarlo fácilmente. Como consecuencia de estas estrategias, \#REme no necesita ser transportado, se pueden variar los materiales adecuándolos a los existentes en la zona y la estructura puede adaptarse a las necesidades del lugar para el que sea requerido.

Durante el desarrollo del primer prototipo, \#REme ha sido expuesto y utilizado en un espacio expositivo popular con el fin de concienciar a los asistentes de la grave situación que sufren actualmente los refugiados y desplazados que huyen de la guerra. De esta manera, este primer prototipo ha servido como espacio expositivo, como zona de talleres de concienciación infantil, y como testeo de la reacción social ante un espacio singular, llamativo y jovial para informar a la población de una situación crítica que supera nuestras capacidades de entender la dimensión de un conflicto bélico.

Es creciente el uso de estructuras de fabricación digital, performances o actuaciones arquitectónicas temporales para la concienciación y la comunicación. Las tecnologías digitales no sólo expanden el campo de la comunicación hacia Internet si no que redefinen la comunicación en el espacio físico. La maquinaria intrínseca de los espacios de autoproducción digital permite que las acciones singulares a través de técnicas BTL (Below the Line) (del Campo, 2002) adquieran nuevas formas de expresión a través de la arquitectura.

Este artículo, muestra cómo un espacio arquitectónico como es un refugio temporal, se puede convertir en una herramienta de comunicación y concienciación muy potente. Así, los factores estrictamente arquitectónicos como son el sistema constructivo, su capacidad espacial, estética o su capacidad para albergar diferentes usos son agentes de mediación de gran valor y potencia divulgativa. Es decir, la estructura arquitectónica amplía su misión y se convierte en un medio de comunicación de valores y puntos de vista, amplificando su capacidad comunicadora. De esta manera, a través de acciones concretas de comunicación BTL la arquitectura se convierte en centro de la acción comunicativa.

\section{2. \#REme}

\#REme es un refugio temporal diseñado a partir de un sencillo sistema constructivo. Se basa en una unidad estructural, hexágono, realizada a partir de dos piezas de pequeñas dimensiones: una que define el lado del polígono y otra que hace de unión entre los 
lados del mismo. A partir de la repetición de esta figura se produce una estructura estable comúnmente definida como panal de abeja. Estas estructuras son las que definen tanto las paredes como la cubierta del refugio. Dado el pequeño tamaño de las piezas y la sencillez del sistema, esta estructura principal se puede realizar en casi cualquier material que tenga un mínimo de resistencia (madera, plástico, panel de cartón...), dependiendo su ejecución de los materiales y tecnología que se disponga.

El aislamiento térmico y acústico se consigue mediante un sistema de membrana inflable de látex, poliamida o similar. Finalmente hace falta una capa final de material textil de poliamida tipo kevlar, para que el refugio sea impermeable además de resistente al punzonamiento. Esta fase del proyecto es la que está en vías de desarrollo.

Figura 1. Piezas del sistema lego que configura el refugio \#REme. Fuente: elaboración propia

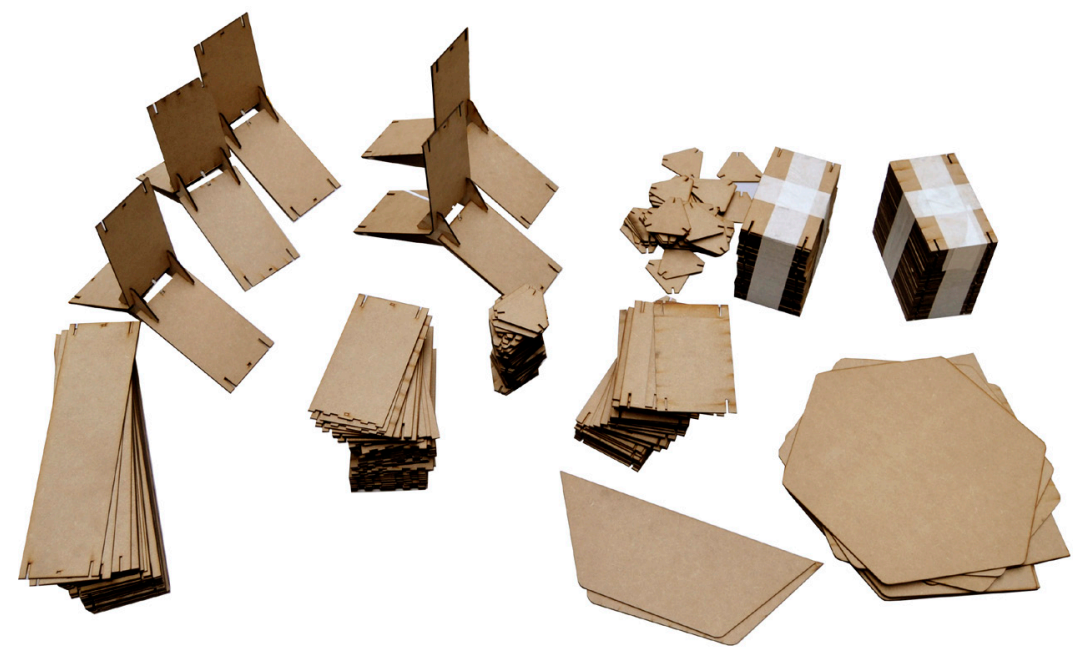

\subsection{LEGO vs IKEA}

La autoconstrucción es una práctica arquitectónica en la que un usuario o grupo de aficionados realizan un conjunto de tareas que normalmente son desarrolladas por varios profesionales o especialistas. Este tipo de práctica en la que se sustituye el profesional u operario cualificado por personal amateur (Basar, 2009), se suele dar en dos tipos de situaciones. La tradicional y popularmente más extendida es aquella en la que una persona, familia o colectivo construyen mediante este método ante la falta de recursos económicos. La más contemporánea y reciente es aquella en la que diseñadores, arquitectos o investigadores realizan dicha práctica con el fin de experimentar y testear posibles soluciones o nuevas herramientas.

LEGO (LEGO, 2017) es un juego de construcción infantil basado en el encaje de pequeñas piezas de plástico que mediante una unión machihembrada permite construir infinidad de juguetes como castillos, barcos, casas o cohetes. LEGO no es el único sistema de autoconstrucción popular en el que es necesario la implicación del usuario para llegar a un resultado final. IKEA por ejemplo basa el éxito de su expansión y crecimiento como 
empresa justamente en un sistema de este tipo. Los productos que ofrece esta tienda de muebles parten de dos estrategias que permiten abaratar sus costes. El primero es el autoservicio del producto por parte del cliente, que va a la tienda y elige lo que desea como si de un supermercado se tratara. La segunda y más innovadora es la que implica al usuario en el montaje de sus muebles, ya que estos están embalados para optimizar su transporte y que posteriormente se proceda a su construcción en el propio hogar o lugar de destino.

LEGO e IKEA son dos formas de resolver problemas de construcción muy diferentes. Basándonos en el concepto de Simon hablaríamos de la diferencia entre la búsqueda la solución óptima -IKEA- y la búsqueda de la solución satisfactoria -LEGO. Esta es la diferencia principal entre análisis y diseño. Y según el problema será más apropiado un método u otro (Simon, 1969).

IKEA basa su concepto de autoconstrucción principalmente en la estrategia del ensamblaje. Es decir, se unen diferentes piezas mediante un número limitado de anclajes o tornillos que no suelen necesitar de más de una o dos herramientas, como pueden ser destornillador y una suerte de llave allen propia. LEGO, en cambio basa su famoso juego de construcción en una pieza modular con una única manera de ser unida, pero que puede variar en dimensiones y colores. Esta variación en los dos sistemas de construcción tiene sin embargo resultados muy alejados entre sí. Con su sistema, IKEA impone una única manera de montaje unidireccional, asegurando así un único resultado final en forma, color y calidad buscando la fidelidad a lo ofrecido por su catálogo de muebles, imponiendo un criterio de homogeneidad y calidad estética desarrollada en el imaginario colectivo a través de su catálogo anual. LEGO, sin embargo, lo que ofrece es un sistema abierto de piezas con la que construir un mundo infinito de objetos, formas y resultados basados en un único sistema de unión. Esto permite utilizar LEGO como base para el aprendizaje y el desarrollo colaborativo a través del diseño (Resnik y Ocko, 1990). Y nos permite llegar a la solución óptima de diseño ya que permite adaptar la construcción a las restricciones y problemáticas particulares de cada emplazamiento.

\#REme es principalmente un sistema constructivo basado en el mismo concepto que aporta LEGO, aplicado al diseño. Se trata de un sistema basado en la simplicidad de su ensamblaje mediante dos piezas principales que configuran una unidad estructural. El resultado formal sin embargo no es único ni unidireccional ya que permite una infinidad de formas finales posibles, siendo el refugio solo el primero de ellos. El sistema permite así que tanto el tamaño, como la forma final del habitáculo y evidentemente su uso, dependan de los recursos económicos, capacidad técnica e intereses del usuario-productor amateur.

\section{Mundo Fab Lab y fabricación digital}

No sois bienvenidos entre nosotros. No ejercéis ninguna soberanía sobre el lugar en el que nos reunimos.

Barlow, (1996)

Este proyecto se basa en los principios básicos que rigen la actividad de cualquier Fab Lab del mundo y que no son otros que los mismos que dieron origen a finales de los años 60 del siglo pasado a Internet. La Red fue creada por un grupo de inves- 
tigadores y académicos, autodenominados hackers, defensores de los valores de la distribución del conocimiento científico: tiene que ser abierto, compartido, revisable y jerarquizado por meritocracia (Tascon y Quintana, 2012).

Estos principios a su vez, junto al desarrollo tecnológico e ideológico generado alrededor de la invención del ordenador personal (1973), tenían en común una filosofía de trabajo que compartían la mayoría de científicos y desarrolladores de la época y que estaba ligada a la propia lógica de los ordenadores. Todo ello ayudó a definir lo que se conoce como ética hacker (Levy, 1984), que además de los principios anteriormente citados incluye: acceso ilimitado a los ordenadores y cualquier fuente de conocimiento, información totalmente libre, la descentralización como estrategia ante la desconfianza por la autoridad y el beneficio común por encima de cualquier interés privado, económico o político.

\subsection{Código abierto: replicabilidad, mutabilidad y usos múltiples}

El refugio \#REme tiene uno de sus pilares en la cultura open source o código abierto. Concepto, que actualmente, ha trascendido al mundo de la informática y el software. Se trata de una nueva manera de entender el concepto de propiedad alrededor de la libertad de distribución y no exclusión (Weber, 2004). La colaboración, el trabajo libre y abierto permite crecer el proyecto, ya que supero el concepto de autoría en pos del de comunidad. Al contrario que ocurre tanto con el sistema IKEA como con el sistema LEGO, \#REme huye de patentes y derechos de autor para ofrecer su sistema en forma de licencia abierta Creative Commons. Se trata de un sistema que basa su fortaleza en su capacidad de replicabilidad, mutabilidad y en todas las alteraciones o variaciones que cualquier usuario, diseñador o productor amateur pueda aportar. Con ello se pretende permitir que el diseño y soluciones que aporta \#REme puedan ser implementadas y amplificadas por la comunidad, con la única condición de que todas las nuevas variaciones y versiones del sistema sean devueltos a la comunidad de la misma manera, en forma abierta y liberada de cualquier restricción legal para su uso y desarrollo.

Esta posibilidad de ser ampliado y transformado está directamente relacionada con su capacidad de usos múltiples. Al tratarse de un sistema constructivo, \#REme no tiene por qué ser un refugio, sino que se puede convertir en aula provisional, casa de juegos infantiles, almacén, caseta temporal, invernadero, o coger otras derivas en las que el espacio habitable no es requerimiento, como celosía, mampara, biombo, falso techo, muro técnico, fachada vegetal o probeta para ensayos biotecnológicos.

\subsection{Descentralización: la red fab labs}

\#REme se basa en la fabricación digital, que consiste principalmente en llevar los conceptos básicos de la revolución digital a la producción de objetos. Los procesos de fabricación de casi cualquier producto hoy en día se basan en la cadena de producción impuesta por el fordismo. Se trata de un proceso lineal que habitualmente responde a una cadena cuyo principal objetivo es la eficacia y rendimiento entendiendo ésta desde el punto de vista de la rentabilidad económica. Un proceso que habitualmente tiene 3 fases:
Ideación
Diseño
Producción 
Durante la primera de ellas, se detecta un problema o se plantea un reto a resolver. Suele ser una fase más o menos abierta en la que una persona o equipo tratan de dar forma a una serie de conceptos que puedan aportar posibles soluciones o innovaciones que resuelvan o mejoren situaciones reales. La segunda fase corresponde al diseño del producto propiamente dicho. Habitualmente es el mismo equipo que el de la primera fase, pero implementado con expertos en las diferentes áreas que fueran necesarias dependiendo del tema y las necesidades del proyecto (expertos en materiales, cálculo de estructura, instalaciones, ecólogos, sociólogos, geógrafos, etc.). La tercera y última fase corresponde a la producción y habitualmente está desarrollada por un sector distinto al de las dos fases anteriores. Así pues, dependiendo de si se trata de una construcción o un producto, entrarían en juego otros agentes como la industria o las empresas constructoras, que serían las únicas capaces de producir y realizar cualquier diseño en serie y en grandes cantidades.

La fabricación digital, sin embargo, aporta una innovación notable a este respecto. Rompe la linealidad de ese proceso de manera que la persona o equipo de trabajo puede y en la mayoría de casos lo es, ser exactamente el mismo. De esta manera, y uniendo ideación, diseño y producción en un mismo equipo y espacio se rompe literalmente esa cadena. Ya no es necesario seguir un protocolo más o menos lineal, sino que se puede pasar de la ideación a la producción de piezas para ver si tiene algo de sentido lo que se está proponiendo, para a continuación pasar al diseño y que éste permita replantearse los conceptos iniciales y así podríamos seguir de una forma rizomática y no lineal del proceso.

Ideación $<>$ Diseño $<>$ Producción

Figura 2. Fabricación digital como proceso no lineal. Fuente: elaboración propia

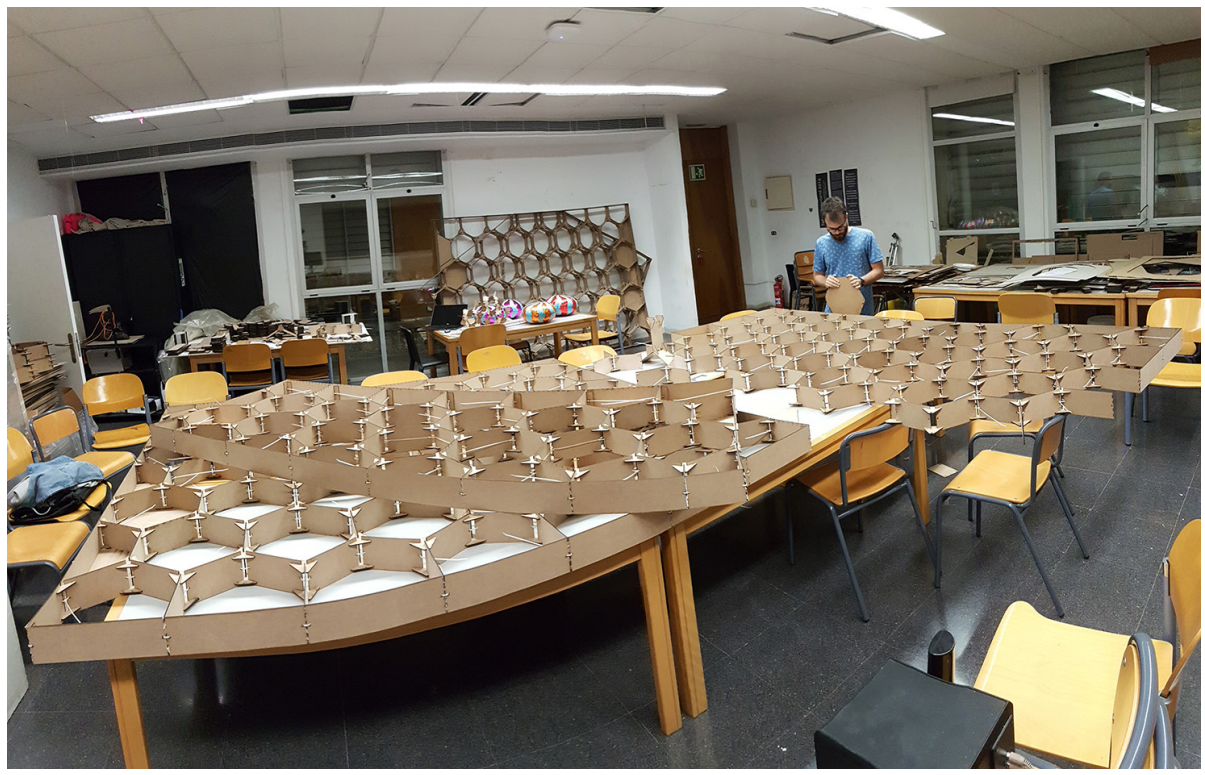


Esta aportación básica para entender el funcionamiento y dinámica de cualquier fab lab o maker space del mundo, tiene otra afección importante a destacar. Se trata de la descentralización. La no linealidad de la cadena de producción fordista a la que el capitalismo nos tiene acostumbrados, se ve de nuevo alterada o puesta en entredicho por este otro concepto. Los fab labs y maker space forman por su propia idiosincrasia una red internacional de centros de producción digital capaces de replicar y fabricar casi cualquier producto o sistema diseñado y desarrollado en cualquier lugar del mundo.

\#REme basa su efectividad como estrategia de refugio para situaciones de emergencia, justamente en la descentralidad que la red internacional aporta. La posibilidad de ser fabricado en cualquier centro de producción digital del mundo permite pensar en su eficacia para ser exportado a cualquier lugar para el que sea necesario un habitáculo de estas necesidades, para ser construido con la tecnología y materiales locales, así como adecuar sus costes a los propios del lugar, evitando así aranceles, sobrecostes y promoviendo la economía local.

\subsection{Tecnología como infraestructura y conocimiento como empoderamiento social}

Otro de los valores que explora \#REme desde su concepción es la inclusión de la tecnología como infraestructura en situaciones de emergencia como pueden ser un terremoto o una crisis humanitaria. Se ha convertido en una práctica habitual ver como las agencias de ayuda humanitaria han desarrollado una logística capaz de montar hospitales de campaña en menos de 24 horas y campos de refugiados de primeras necesidades en apenas 48 horas.

La propuesta que se aporta desde la concepción del refugio consiste en utilizar la logística propia de estas situaciones no para llevar soluciones provisionales como son un refugio prefabricado, sino máquinas de corte, fresadoras y la tecnología básica de un centro de producción digital. Esta tecnología se convertiría así en una infraestructura capaz de producir in situ soluciones viables de bajo coste como puede ser \#REme o cualquier otro prototipo o dispositivo necesario y previamente desarrollado en cualquier parte del mundo. Esta situación todavía no ensayada, permitiría que la tecnología aportada como solución de emergencia se convirtiera en infraestructura para futuros desarrollos de nuevos proyectos. Es decir, se trata de aportar tecnología y conocimiento en lugar de soluciones y paliativos temporales.

Por otro lado, el conocimiento aportado y aprendido por las personas/sociedades/grupos locales ayudaría no solo al desarrollo de nuevos proyectos, sino que estaría generando empoderamiento social. Frente a la actitud pasiva y de dádiva con la que se suele estigmatizar y minusvalorar a las comunidades que han sufrido una crisis, con esta revisión de los protocolos de ayuda humanitaria, las personas y comunidades afectadas pasarían de un estado pasivo de recepción de ayuda a un estado activo de colaboración. Se trataría de entender a esos damnificados como productores locales, capaces de ayudar a gestionar una crisis como cualquier voluntario, cooperante o agente experto. 


\section{Ensayo}

Este primer ensayo se realizó en el contexto de un mercado navideño temporal que duró tres días en un espacio público y abierto de Alicante. Tras la difusión del primer prototipo de refugio temporal, una ONG local solicitó exponer el dispositivo para realizar una campaña de concienciación sobre la situación de los migrantes sirios en los campos de refugiados griegos.

Esta campaña implicaba varios usos y significados distintos a través del uso del refugio. Por un lado, era un espacio amable, colorista y atractivo con el que atraer y dar cabida a una serie de actividades lúdico-infantiles de concienciación social: talleres de pintura y construcción de maquetas, conciertos y espacio expositivo. Con estas acciones se perseguía concienciar desde un punto de vista optimista a los ciudadanos sobre una situación de riesgo y exclusión social, que son mostradas habitualmente desde el miedo, la miseria y la desesperación.

Figura 3. Refugio como taller infantil de pintura. Fuente: elaboración propia

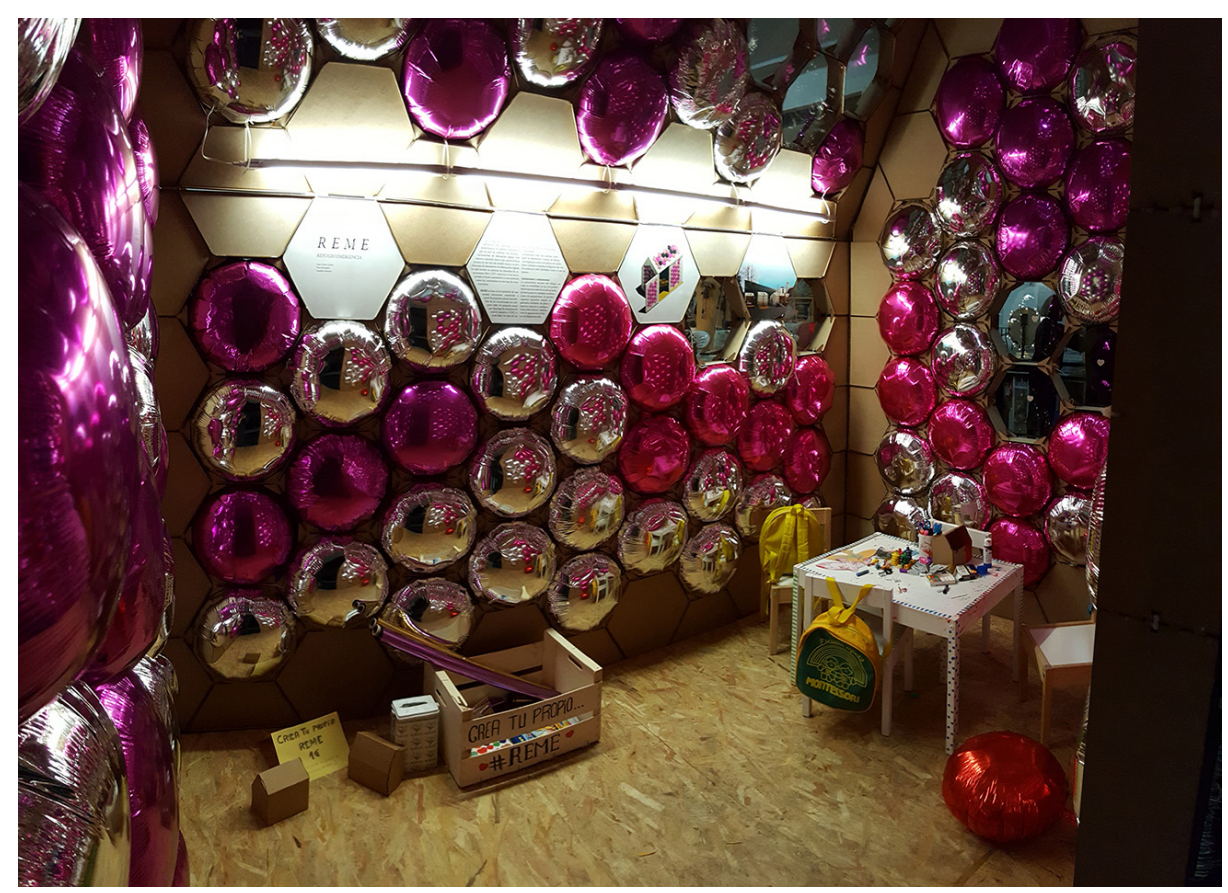

Por otro lado, servía como modelo de posibles soluciones temporales vinculadas a la infancia como podría ser un aula infantil o un espacio lúdico para campos de refugiados. Se trataba de exponer públicamente la posibilidad de construir un espacio de fácil ejecución y bajo coste y con ello demostrar que el soporte económico solidario con este tipo de causas no se diluye en grandes operaciones de difícil visibilidad, sino que por el contrario pueden llegar a materializarse en elementos y acciones tangibles que se pueden conseguir desde la acción de micro grupos o colectivos pequeños.

Este primer ensayo permitió testear la importancia de la experiencia espacial de los dispositivos arquitectónicos como agentes generadores de conocimientos alternativos 
al de la propia práctica arquitectónica (Miessen, 2014). No se trata tan sólo de entender la arquitectura como productora de espacios habitables, y constituyente de teoría y conocimiento arquitectónico, sino que su propia práctica puede y constituye otras formas de conocimiento y comunicación alternativos en el ámbito de las instituciones. Agentes altamente tecnológicos y especializados como pueden ser el sistema constructivo, la ejecución de los mismos, la construcción de membranas neumáticas o la transformación de elementos comunes en elementos constructivos mediante el uso de técnicas populares como la autoconstrucción y tecnologías en boga como la fabricación digital, se muestran en este ensayo como agentes de capaces de atraer la atención e interés de la ciudadanía que descubren mediante este ejemplo posibilidades de construcción reales, asequibles y cercanas tanto cultural como económicamente.

Figura 4. Evento público. Fuente: elaboración propia
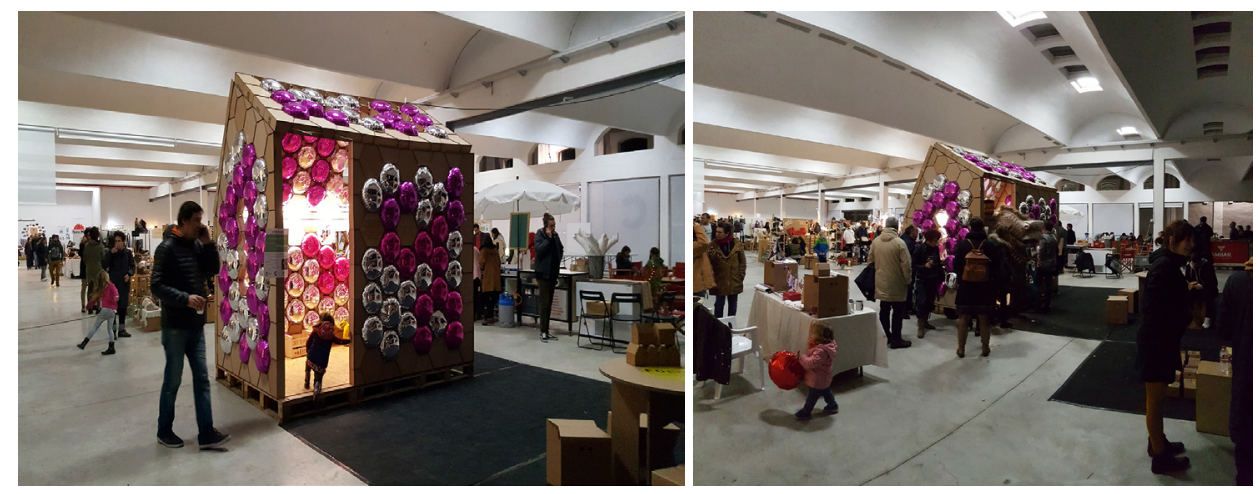

\section{Arquitectura para la comunicación semiótica y diseño}

Este tipo de construcciones están cargadas de connotaciones negativas ya que se llega a ellos a través de situaciones de emergencia, bien sea una catástrofe natural, una guerra o una revolución social violenta. Así no es de extrañar que al pensar en un refugio para situaciones de emergencia uno se imagine una tienda de campaña, una nave industrial, una chabola o una estructura gris más similar a un contenedor de puerto que a una casa. Sin embargo, estas situaciones que pretenden ser temporales son parte de la vida de miles de personas y por lo tanto se pueden construir diferentes soluciones y maneras de enfrentarse a estas situaciones. Aunque el fin último de \#REme es ser una herramienta de empoderamiento, el objetivo de nuestra acción durante el ensayo era cambiar la percepción de esta etapa en la que se necesita más esperanza y recordar aquello que nos une, nos hace humanos y felices.

En este contexto analizamos \#REme a partir del desarrollo semiótico de la comunicación llevado a la construcción no de la palabra o la imagen si no de la estructura arquitectónica. Y es a través de esta construcción como medio de comunicación del mensaje a través de la que se pretende cambiar las ideas preconcebidas. Se pretende convertir al arquitecto en mediador de la comunicación, en constructor de sociedades en momentos donde es necesaria la colaboración a través del diseño y al usuario en parte del proceso de construcción de significado. Para un diseño basado en valores 
partimos de la idea macluhiana de que el medio es el mensaje y entre ambos existe una relación simbiótica.

Desde la concepción semiótica de Peirce del referente (la entidad en el mundo real), un refugio inscrito en un campo de refugiados durante una situación de emergencia, hemos intentado a través de la modificación del referente, una disrupción del signo (la figura) para conseguir que el sujeto se replantee su significado (la interpretación que hace del signo el observador) (Redondo Domínguez, 2009). Este proceso es habitual en arquitectura y puede ser muy útil como herramienta de comunicación social. Así partimos de signos asociados a otros referentes para modificar nuestro objeto haciendo que cambie el significado para el sujeto final y se construya un nuevo signo.

El método es definir nuevos significados y asignar a \#REme, prototipo de refugio para situaciones de emergencia, estos significados y connotaciones positivas a través de la forma de aproximación al problema de la mente del arquitecto. Así la metodología tradicional del diseño arquitectónico se une a las nuevas formas de producción en la búsqueda de nuevas aproximaciones a problemas sociales. Para definir estas nuevas significaciones y connotaciones se ha hecho a través de la herramienta principal del arquitecto: el diseño, y se le ha dado forma y se ha realimentado con herramientas de fabricación digital, permitiendo así que la forma de producción y construcción formen parte de la metodología y exista una relación simbiótica entre el proceso y el objetivo.

Partimos de refugio como hogar temporal. El diseño nos permite dar una forma hogareña a la que podamos asignar valores positivos como el de pertenencia o confort. Las edificaciones con tejados a dos aguas son formas icónicas asociadas al hogar en occidente. Es habitual que los niños dibujen casitas con este tipo de tejados. El diseño nos permite asociar las connotaciones de este signo a nuestra construcción. Esto nos abre todo un campo semántico positivo y empoderador que será el sujeto quien lo rellene.

El siguiente paso es respuesta a una situación de emergencia como respuesta de la comunidad. Las estructuras hexagonales del diseño se pueden asociar al concepto de comunidad, ya que están distribuidas por toda la superficie interactuando entre ellas para generar unidades estructurales. Esta decisión de diseño nos da una nueva capa de significación. El campo semántico de comunidad, con ideas como apoyo, interacción o solidaridad, está implícito en esta decisión de diseño. Además, esta forma habitualmente llamada de panal de abeja nos abre una segunda lectura más orientada hacia la colaboración y la superación.

En esta situación podemos cambiar la percepción si damos la posibilidad de ser útiles y de aportar algo al individuo. La solidaridad tiene que ser sencilla de poner en marcha. Estos dos objetivos se consiguen a través de la fabricación digital, es un mecanismo de empoderamiento, permite al sujeto ser dueño de la construcción de esta estructura ayudado de la comunidad. Aquí proponemos un nuevo campo de significación haciendo al usuario final dueño de su destino. Esta idea está muy arraigada en las sociedades occidentales, donde se valora al ser humano hecho a sí mismo, capaz de superar las dificultades. Y aquí es donde para nosotros nace la idea de optimismo que finalmente se convertirá en centro del diseño.

El optimismo es algo que no se debe perder en las situaciones difíciles por lo tanto hemos querido asociar esta connotación al diseño. Uno de los principales vehículos de transmisión de connotaciones es el color, por eso se ha optado por el rosa.

Quien opta el lema think pink se propone vivir de manera optimista la gris cotidianidad. Cuando la vida es como un sueño, dicen los franceses: C'est la vie en rose. [...] Color de la transfiguración (Heller, 2004, p. 218). 
Pero no cualquier rosa, un rosa poderoso, llamativo y fuerte. Un rosa desinfantilizado que toma su fortaleza y su valor del rojo, agresivo, pasional, atractivo y lujoso (Heller, 2004) para que su efecto cromático y por tanto sus connotaciones dominen sobre lo demás.

Despojamos al rosa de su infancia y dotamos al individuo de herramientas para manejar su futuro, estas dos herramientas de diseño unidas despojan a la situación de esta connotación socialmente paternalista de cuidado al desvalido. A través del análisis arquitectónico proponemos una nueva solución a un problema que lleva mucho tiempo estancado.

Finalmente es una estructura adaptable que permite personalización y adaptación a las necesidades de una manera relativamente sencilla. Estos valores quedan adheridos a los anteriores. Son connotaciones más en consonancia con la arquitectura actual, asociados a los millenials y las nuevas tecnologías, que nos permiten una revisión de las maneras tradicionales de enfrentar los problemas. Nos permiten proponer una disrupción a las ideas tradicionales y presentar nuevas realidades que cambian al signo y lo enriquecen.

\subsection{Disrupción en el objeto arquitectónico}

El evento también permite exponer el análisis semiótico de la comunicación arquitectónica. Pretendíamos mostrar como cambiando el objeto arquitectónico a través de connotaciones cambiamos el enfoque y llegamos a diferentes significados y por tanto diferentes maneras de aproximarse a un problema.

Todo este trabajo ha permitido que \#REme actúe como centro de atención del mensaje. Hemos cambiado el discurso. Nos ha permitido hablar de infancia, de la temporalidad que debería ser una característica de estas construcciones y que normalmente no lo es, del paternalismo social e institucional, pero sobre todo nos ha permitido hablar de solidaridad, de comunidad, de optimismo, de hogar o de comodidad. \#REme nos ha permitido romper con los estereotipos y empezar la conversación para modificar una realidad.

Figura 5. Arquitectura como dispositivo lúdico-festivo de comunicación. Fuente: elaboración propia

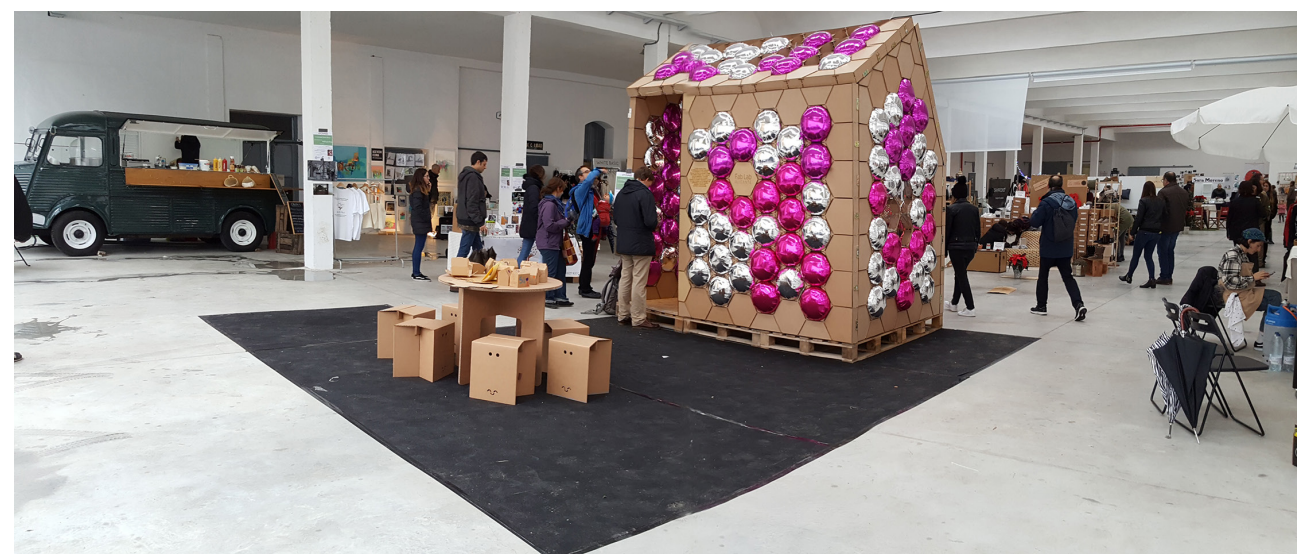


Durante el evento \#REme se transformó en un lugar de ocio, de comunidad, de solidaridad, de pensamiento y de esperanza. Nuestra aportación de campos semánticos fue inundada por la acción de otros sujetos que añadieron sus perspectivas y sus vivencias. Pero sobre todo fue un giro positivo a la comunicación de estas circunstancias. A veces, ante situaciones difíciles se pierde el optimismo y la esperanza, un cambio en el espacio como es \#REme impulsa un cambio en el mensaje, ya que en las acciones BTL el espacio interactúa de manera simbiótica con el mensaje.

Un espacio como \#REme atrae.

\section{Conclusiones}

Arquitectura como acción disruptiva en la que rompe con el estereotipo de refugio como espacio temporal, ajeno al optimismo y al valor lúdico como recurso para el empoderamiento social. Se trata de una nueva manera de mirar que aporta nuevas connotaciones al concepto de refugio.

Uso de la experiencia de la construcción como un nuevo inicio para el uso de la arquitectura como medio de comunicación desde nuevas práctica espaciales, experienciales y constituyentes. Se trata de reformular el significado a partir del medio -experimentación, prototipado y autoconstrucción.

Valor de la autoproducción como empoderamiento social y político. El diseño se convierte en una herramienta de aportación de significado al objeto que obliga al individuo a reconstruir el signo para dar cabida a nuevos campos semánticos.

\section{Referencias}

Barlow, J.(1996). Declaración de Independencia del Ciberespacio. Disponible en: http://www. uhu.es/ramon.correa/nn_tt_edusocial/documentos/docs/declaracion_independencia.pdf

Belloc, H. (1967). On. Freeport, N.Y.: Books for Libraries Press.

Berger, J. (2016). Modos de ver. 3rd ed. Barcelona: Editorial Gustavo Gili.

Del Campo, E. P. (2002). Comunicación fuera de los medios: "below the line”. Madrid: ESIC

Heller, E. (2004). Psicología del Color: Como actúan los colores sobre los sentimientos y la razón. Barcelona: Editorial Gustavo Gili.

Levy, S. (1984). Hackers. Heroes of the computer revolution. New York: Dell.

Miessen, M. (2014). La pesadilla de la participación. Barcelona: DPR-Barcelona.

Miessen, M. and Basar, S. (2009). ¿Alguien dijo participar? Barcelona: DPR-Barcelona.

Nascimento, S., Pólvora, A., Paio, A., Oliveira, S., Rato, V., Oliveira, M., Varela, B. and Sousa, J. (2016). Sustainable Technologies and Transdisciplinary Futures: From Collaborative Design to Digital Fabrication. Science as Culture, 25(4), 520-537.

Redondo Domínguez, I. (2009). El signo como medio: Claves del pensamiento de C.S. Peirce para una teoría constitutiva de la comunicación. Tesis doctoral.

Simon, H. (2008). The sciences of the artificial. Cambridge, Mass.: MIT Press.

Tascón, M. y Quintana, Y. (2012). Ciberactivismo. Madrid: Catarata.

Weber, S. (2005). The success of open source. Cambridge, Mass.: Harvard University Press. 